\title{
The wide world of rheumatology
}

\author{
Georg Stummvoll
}

Published online: 4 November 2016

(C) Springer-Verlag Wien 2016

What a wide range of symptoms and diseases can be seen in rheumatology outpatient wards: autoimmune diseases, such as rheumatoid arthritis and psoriatic arthritis with their proinflammatory responses and diseases, such as common variable immunodeficiency brought about by a lack of key immunological components. There are also conditions with difficult differential diagnostics in the large overlapping field of infections and rheumatology, presenting as fever of unknown origin or as infections leading to neurological problems, e.g. peripheral facial nerve palsy as a sign of neuroborreliosis, with the potential to also affect the musculoskeletal apparatus. There are complex clinical features that result from inflammation of blood vessels and there is the challenge of using and interpreting correct imaging techniques, illustrated in this issue of the Journal by an article on imaging findings in pulmonary granulomatosis with polyangiitis (GPA, formerly known as Wegener's granulomatosis) and by case reports on large vessel arteritis and one on acute myocardial infarction as a manifestation of systemic vasculitis. The complex situation of differential diagnostics and the challenges it brings is illustrated by a case of psoriasis and arthralgia discussed in the Clinical Pathological Conference Series from the Medical University of Graz.

In daily practice and in clinical science, rheumatology is also closely related to rehabilitation. In this issue, the topic of neuromuscular electrical stimula- tion in patients with implantable cardioverter defibrillators is highlighted, as is the evaluation of sarcopenia in patients suffering from fibromyalgia syndrome, a still unclear disease with pain of unknown origin. Pain, inflammation and infections are also the main issues in orthopedic surgery (e.g. after femoral fractures) and new techniques for dealing with these are being investigated. Osteoporosis as a potential cause of these fractures is also one of the main comorbidities found in inflammatory rheumatic diseases. These comorbidities are increasingly becoming the focus of scientific interest with each step forward representing progress in the treatment of the acute inflammatory disease itself.

The aim of this issue of "Wiener Klinische Wochenschrift - The Central European Journal of Medicine" is to provide an overview of the wide field of current topics in rheumatology research for specialists in all areas of (internal) medicine and to present interesting articles for rheumatologists.

I hope that this first "Rheumatology Issue" will please our readers and stimulate interest in articles on related topics coming soon in the Journal.

Conflict of interest G. Stummvoll declares that he has no competing interests.

\footnotetext{
G. Stummvoll, MD, Associate Professor $(\bowtie)$

Department of Internal Medicine III, Division of

Rheumatology, Medical University Vienna, Währinger Gürtel

18-20, 1090 Vienna, Austria

georg.stummvoll@meduniwien.ac.at
} 